\title{
Medidas de conservación y ordenación pesquera del jurel Trachurus murphyi en el Perú
}

\section{Conservation and fishery management regulations of Jack mackerel Trachurus murphyi in Peru}

Instituto del Mar del Perú, esquina Gamarra y General Valle S/N Chucuito, Callao, Perú.

Email Jorge Zuzunaga: jzuzunaga@imarpe.gob.pe
Citación:

Zuzunaga J. 2013. Medidas de conservación y ordenación pesquera del jurel Trachurus murphyi en el Perú. En: Csirke pesquera de ju J., R. Guevara-Carrasco \& M. Espino (Eds.). Ecología, pesquería y conservación del jurel (Trachurus murphyi) en
el Perú. Rev. peru. biol. número especial 20(1): 107 - 113 el Perú. Rev. peru.
(Septiembre 2013)
Jorge Zuzunaga

\begin{abstract}
Resumen
Las pesquerías peruanas como actividades realizadas sobre recursos naturales renovables son reguladas por un marco jurídico, cuyo objetivo es asegurar su conservación y uso sostenible. La Ley General de Pesca (DL N․ 25977) señala que para cada tipo de pesquería y según la situación del recurso explotado debe establecerse un sistema de ordenamiento que concilie el principio de sostenibilidad de los recursos pesqueros o conservación en el largo plazo, con la obtención de los mayores beneficios económicos y sociales. En el ordenamiento pesquero del jurel Trachurus murphyi, las medidas incluyen entre otras, el régimen de acceso constituido por las autorizaciones de incremento de flota y permisos de pesca, que se otorgan solo a los buques cuyas capturas son destinadas únicamente para el consumo humano directo, de acuerdo a lo dispuesto en el Reglamento de Ordenamiento Pesquero (DS N. ${ }^{\circ}$ 011-2007-PRODUCE). La obligación de destinar los recursos sardina Sardinops sagax, jurel T. murphyi y caballa Scomber japonicus al consumo humano directo rige desde septiembre 2002 (DS Nº 001-2002-PRODUCE). Otras medidas vigentes restringen el tamaño mínimo de malla de las redes, prohíben la captura de ejemplares menores de $31 \mathrm{~cm}$ de longitud total, determinan el inicio y cierre de las temporadas de pesca, los límites de captura o cuotas anuales. Estas disposiciones son complementadas por medidas de seguimiento, control y vigilancia para la efectiva conservación y uso sostenido del recurso.
\end{abstract}

Palabras clave: Conservación, ordenación, jurel, pesquerías

\section{Abstract}

The Peruvian fisheries exploit renewable natural resources and as such are regulated by a legal framework aiming at ensuring their conservation and sustainable use. The General Fisheries Act, DL N. 25977, points out that fisheries management systems shall be established for each type of fishery taking into account to the state of exploitation of the fishery resources, reconciling the principles of sustainability and long-term conservation with those aiming at the attainment of the maximum possible economic and social benefits. According to the provisions of the Fisheries Management Regulations approved by SD N ${ }^{\circ}$. 011-2007-PRODUCE the management of the Jack mackerel Trachurus murphyi fisheries includes among others, a fishing access regime that regulates the fleet size and the allocation of fishing licenses, which can only be granted to vessels fishing only for direct human consumption. The obligation to allocate the resources sardine Sardinops sagax, Jack mackerel T. murphyi, and mackere Scomber japonicus, for direct human consumption has been in force since September 2002, following the ruling established by SD N. 001-2002-PRODUCE. Other measures currently in force include those establish a minimum mesh size, the ban to catch or land specimens under $31 \mathrm{~cm}$ total length limit, the opening and closing of the fishing seasons, and catch limits or annual quotas. These provisions are supplemented by measures for monitoring, control and surveillance, and for the effective conservation and sustainable use of the fishery resources.

Keywords: Conservation, management, Jack mackerel, fisheries 


\section{Introducción}

El Perú ha adoptado un marco normativo jurídico e institucional que establece medidas para la conservación y el uso sostenible a largo plazo de los recursos pesqueros. Este marco normativo se inspira y está plenamente en concordancia con los principios rectores del Código de Conducta para la Pesca Responsable (FAO 1995). Es así que en la legislación peruana se establecen medidas de conservación y ordenación, aplicables al aprovechamiento de los recursos naturales en general (Ley $\mathrm{N}^{\circ}$. 26821) y pesqueros en particular (DL N. 25977, Ley General de Pesca).

En principio, la Ley General de Pesca norma esta actividad con el fin de promover su desarrollo sostenido como fuente de alimentación, empleo e ingresos y de asegurar un aprovechamiento responsable de los recursos hidrobiológicos, optimizando los beneficios económicos, en armonía con la preservación del medio ambiente y la conservación de la biodiversidad. Pero a su vez, las medidas de ordenamiento deben basarse en los datos científicos más rigurosos disponibles y deben estar diseñadas para garantizar la sostenibilidad a largo plazo de los recursos pesqueros.

En el presente trabajo ofrecemos una breve descripción de las medidas de gestión aplicables a los stocks peruanos del jurel Trachurus murphyi Nichols 1920 y de la caballa Scomber japonicus Linnaeus 1758, que como se describe más adelante, para los fines de ordenamiento fueron considerados como partes de una misma pesquería, en razón de encontrarse frecuentemente distribuidos en las mismas áreas y por lo tanto, ser accesibles a las mismas flotas y artes de pesca.

\section{Marco legal e institucional}

La Constitución Política del Perú establece los principios que rigen la propiedad y el acceso a la explotación de los recursos naturales. Así, los recursos naturales renovables y no renovables son patrimonio de la Nación y el Estado es soberano en su uso (Artículo $66^{\circ}$ ). También dispone que las condiciones sean establecidas por ley orgánica, para su uso por particulares. Asimismo, el Artículo $67^{\circ}$ declara que el Estado determina la política nacional del ambiente y promueve el uso sostenible de los recursos naturales.

Sobre la base de este mandato constitucional la Ley sobre el Uso Sostenible de los Recursos Naturales Renovables (Ley N. ${ }^{\circ}$ 26821) establece el marco general para promover y regular la sostenibilidad, persiguiendo un equilibrio dinámico entre el crecimiento económico, la conservación de los recursos naturales y el medio ambiente. De acuerdo con este principio, las condiciones de acceso para cada recurso deben ser reguladas por leyes especiales. En el caso de los recursos pesqueros, la misma norma dispone que la Ley General de Pesca (DL Nº. 25977) conserve su plena vigencia, a pesar de que fue dictada con anterioridad a la Ley sobre el Uso Sostenible de los Recursos Naturales.

En este orden de ideas, las disposiciones contenidas en la Ley General de Pesca constituyen el marco jurídico adecuado para regular la actividad pesquera y la promoción de su desarrollo sostenible, mientras que el Reglamento de la Ley General de Pesca (DS Nº 012-2001-PE) establece normas para la determinación de principios y medidas reguladoras que han de aplicarse a las actividades de pesca o extracción de los recursos acuáticos vivos. De acuerdo al principio establecido en la ley y en su reglamento, cada pesquería es tratada como una unidad diferenciada (Artículo $12^{\circ}$ DL N ${ }^{\circ}$. 25977), teniendo presente que para definir el nivel de explotación apropiado a los objetivos del ordenamiento se debe delimitar la unidad de población. Tal es el caso del stock de jurel que se distribuye la mayor parte de las aguas jurisdiccionales del Perú y del cual se ocupa la ordenación pesquera como una unidad de población independiente a la cual se administra de manera sostenible, en el presente caso mediante las disposiciones del Reglamento de Ordenamiento Pesquero de Jurel y Caballa (DS Nº 011 2007). En dicho reglamento se fija como principio el objetivo de promover el aprovechamiento racional de las poblaciones del jurel T. murphyi y la caballa $S$. japonicus, proteger el ecosistema marino y salvaguardar la biodiversidad, contribuyendo a la diversificación y desarrollo de la pesca como fuente de alimento, empleo e ingresos económicos.

Como se observa para el caso de la pesquería de jurel y caballa, el ordenamiento específico de cada pesquería se efectúa mediante reglamentos de ordenamiento pesquero, los cuales en su elaboración son producto de un proceso que toma en cuenta lo que la FAO define como:

“(...) proceso integrado de recolección de información, análisis, planificación, consulta, adopción de decisiones, asignación de recursos y formulación y ejecución, así como imposición cuando sea necesario, de reglamentos o normas que rijan las actividades pesqueras para asegurar la productividad de los recursos y la consecución de otros objetivos" (FAO 1999, p. 7)

Para el efecto, la Ley General de Pesca considera que para establecer el ordenamiento de cada pesquería concurren el conocimiento del recurso y las condiciones de la actividad, señalando que:

“(...) sobre la base de evidencias científicas disponibles y de factores socioeconómicos, se determinará, según el tipo de pesquerías, los sistemas de ordenamiento pesquero, las cuotas de captura permisible, las temporadas y zonas de pesca, la regulación del esfuerzo pesquero, los métodos de pesca, las tallas mínimas de captura y demás normas que requieran la preservación y explotación racional de los recursos hidrobiológicos" (DL No. 25977, Art. 9).

Por lo tanto, el Reglamento de Ordenamiento Pesquero de Jurel y Caballa (DS Nº 011-2007-PRODUCE) contiene las medidas de ordenamiento pesquero para acceder a la actividad extractiva de estas especies, aplicables a las autorizaciones de incremento de flota y permisos de pesca que se otorgan a armadores de embarcaciones de bandera nacional, de cerco, arrastre de media agua, multipropósito (cerco/arrastre de media agua) y de pesca con anzuelo.

\section{Medidas de ordenamiento pesquero del jurel}

En lo que se refiere a la pesca de cerco del jurel, desde 1974 en adelante se establecieron medidas de regulación pesquera como el tamaño de malla, fijándola en $38 \mathrm{~mm}$, y la talla mínima de captura de ejemplares. Desde 1992 (Ley General de Pesca, art. 33; Reglamento de la Ley General de Pesca, DS No. 017-92-PE), con relación a la protección de las áreas de pesca se dispuso la reserva de las primeras 5 millas náuticas $(\mathrm{mn})$ a partir de la línea de costa para la pesca artesanal, por lo que la pesca de jurel por la flota industrial, en cualquiera de sus formas, debía realizarse 
fuera de esta zona.

En la década de los ochenta, la pesca de jurel se compartió entre la flota de cerco y la flota de arrastre factoría, normada por el Reglamento para la Operación de Buques Pesqueros de Arrastre (DS N. 012-84-PE), que establecía que el Ministerio de Pesquería lleve el control de los permisos de pesca conciliando el esfuerzo pesquero con los volúmenes de captura recomendados por el IMARPE. Con el mismo dispositivo, se reservaba para el Estado el 25\% del total de la captura permisible anual de los recursos de jurel, caballa y merluza Merluccius gayi peruanus u otras especies, para cuya explotación podría celebrar convenios con armadores nacionales o extranjeros. Además, normaba el tamańo de malla en $76 \mathrm{~mm}$, restringiendo las áreas de pesca a aquéllas con profundidades no menores a $100 \mathrm{~m}$ y distancia de la costa no menor a $20 \mathrm{mn}$, sin desmedro de las profundidades; es decir, cuando las profundidades eran mayores a $100 \mathrm{~m}$ a una distancia menor de $20 \mathrm{mn}$, la prohibición era aplicada.

En el caso del jurel, el Reglamento de Ordenamiento Pesquero de Jurel y Caballa (DS No. 011-2007-PRODUCE) considera un régimen de acceso restringido sólo a las embarcaciones que cuentan con sistemas operativos de preservación de las capturas a bordo. En relación a esta medida, y como se ha mencionado anteriormente, ya mediante el DS Nº . 001-2002-PRODUCE se estableció que las capturas del jurel deben ser destinadas exclusivamente al consumo directo. Esta norma está vigente desde hace once ańos y viene siendo cumplida a cabalidad.

En el caso de las embarcaciones pesqueras de cerco, el acceso sólo está autorizado cuando sustituye (reemplaza) igual capacidad de bodega de la flota operando con permisos para la extracción de jurel y caballa. Los armadores de las embarcaciones que son autorizadas para realizar actividades pesqueras están sujetos al pago de derechos de pesca y la flota autorizada para extraer jurel y caballa sólo puede operar fuera de las 10 millas de costa. Las embarcaciones de arrastre no pueden operar en áreas con menos de $200 \mathrm{~m}$ de profundidad (DS Nº 011-2007-PRODUCE).

Está determinado un tamaño mínimo de malla para las redes. Para la red de cerco, el tamaño mínimo es de $38 \mathrm{~mm}$ (11/2 pulgadas), y para la red de arrastre de media agua, es de 76 mm (3 pulgadas) en el copo. También se prohíbe la extracción, procesamiento y comercialización de ejemplares de jurel con tallas inferiores a $31 \mathrm{~cm}$ de longitud total y caballa con tallas inferiores a $29 \mathrm{~cm}$ de longitud a la horquilla (equivalente a 32 cm de longitud total).

Complementariamente al reglamento de ordenamiento pesquero, mediante normas específicas, como resoluciones ministeriales, son establecidas las vedas y temporadas de pesca y los correspondientes límites (cuotas) de captura, así como las acciones de monitoreo, control y vigilancia.

El marco actual de ordenamiento de la pesquería de jurel incluye las siguientes medidas y restricciones:

- Tamaño de malla $38 \mathrm{~mm}$ o 11/2 pulgada (red de cerco).

- Tamaño de malla $76 \mathrm{~mm}$ o 3 pulgadas en el copo (red de arrastre).

- Tamaño mínimo de captura: $31 \mathrm{~cm}$ de longitud total.

- Tolerancia de jureles juveniles en la captura: 30\% del número total.
- Reglamento de Ordenamiento Pesquero de Jurel y Caballa (DS Nº. 011-2007-PRODUCE. 2007).

- El uso exclusivo del jurel para consumo humano directo (DS N. 001-2002-PRODUCE).

- Implementación del monitoreo satelital para la flota industrial de acero y de madera (DS N ${ }^{\circ}$. 031-2003-PRODUCE)

- Establecimiento de medidas para la explotación de recursos como el jurel y la caballa, por embarcaciones nacionales de gran escala en la alta mar (DS $\mathrm{N}^{\circ}$. 022-2009-PRODUCE)

- Desde el 2003, la aplicación de cuotas de captura para el jurel por determinados periodos. En este informe se incluye una relación de las normas emitidas en los dos últimos años.

\section{- Principales medidas de ordenamiento y conservación de jurel y caballa en el periodo 2010 - 2012}

A continuación se presenta una relación de las normas de ordenamiento y conservación para los stocks de jurel y caballa, para el periodo más reciente 2010 - 2012. Estas normas han sido dirigidas a establecer los regímenes de pesca y regular las actividades de extracción de estos dos stocks en el marco general del ordenamiento pesquero peruano:

-La RM No. 026-2010-PRODUCE, vigente desde el 3 de febrero del 2010, estableció un régimen provisional para la extracción de jurel y caballa en todo el litoral peruano hasta el 31 de marzo de 2010, de alcance a las capturas provenientes de la actividad pesquera artesanal y de las embarcaciones pesqueras de mayor escala, con permiso vigente para la extracción de dichos recursos.

-La RM No. 027-2010-PRODUCE, vigente desde el 3 de febrero del 2010, estableció un régimen de pesca exploratoria de recursos pelágicos asociados al evento El Niño en todo el litoral peruano, que autoriza la extracción de los recursos bonito (Sarda chilensis), barrilete (Katsuwonus pelamis), melva (Auxis rochei), jurel (T. murphyi) y caballa (S. japonicus) hasta el 31 de marzo de 2010.

-La RM Nº. 096-2010-PRODUCE, vigente desde el 17 de abril del 2010, estableció un régimen provisional para la extracción de jurel y caballa en todo el litoral peruano, hasta el 31 de mayo de 2010, de alcance a las capturas provenientes de la actividad pesquera artesanal y de las embarcaciones pesqueras de mayor escala con permiso de pesca vigente. Establece un límite de captura mensual de $15000 \mathrm{t}$. Una vez cumplido el límite de captura para cada recurso, el Ministerio de la Producción suspendía las actividades extractivas por los días que resten del mes correspondiente. Las actividades extractivas se sujetaron al cumplimiento de las disposiciones del reglamento de Ordenamiento Pesquero de Jurel y Caballa (DS N. 011-2007-PRODUCE).

-La RM N. ${ }^{\circ}$ 097-2010-PRODUCE, vigente del 18 de abril del 2010, estableció un régimen de pesca exploratoria de recursos pelágicos de oportunidad en todo el litoral peruano, asociados al evento El Niño, y que autoriza la extracción de los recursos bonito ( $S$. chilensis), barrilete (K. pelamis), melva (A. rochei), jurel (T. murphyi), caballa (S. japonicus) y 
otros, hasta el 31 de mayo de 2010. Prohibió la extracción, recepción y procesamiento de la anchoveta Engraulis ringens y merluza Merluccius gayi peruanus, incluida su fauna acompañante.

-La RM N. ${ }^{\circ}$ 202-2010-PRODUCE, vigente desde el 18 de agosto del 2010, estableció un régimen provisional para la extracción del jurel $T$. murphyi y caballa $S$. japonicus en todo el litoral peruano por un plazo de sesenta (60) días, de alcance a las capturas provenientes de la actividad pesquera artesanal y de las embarcaciones pesqueras de mayor escala, con permiso de pesca vigente para dichos recursos.

-La RM Nº. 035-2011-PRODUCE, vigente del 6 de febrero del 2011, suspendió las actividades de extracción y recepción del jurel, a partir de las 00:00 horas del 7 de febrero hasta las 24:00 horas del 28 de febrero de 2011, en el marco del régimen provisional de extracción establecido mediante $\mathrm{RM}$ N. ${ }^{\circ}$ 025-2011-PRODUCE.

-La RM Nº . 039-2011-PRODUCE, vigente el 9 de febrero del 2011, modifico los Artículos $1^{\circ}$ y $2^{\circ}$ de la RM N . 035-2011-PRODUCE, del 6 de febrero del 2011 con el siguiente texto: "Artículo $1^{\circ}$.- Suspender a partir de las 00:00 horas del 7 de febrero hasta las 24:00 horas del 28 de febrero de 2011, las actividades de extracción del recurso jurel, en el marco del Régimen Provisional de extracción establecido mediante RM N. ${ }^{\circ}$ 025-2011-PRODUCE. Artículo $2^{\circ}$. - La actividad de procesamiento del recurso jurel podrá desarrollarse hasta las 24:00 horas del 9 de febrero de 2011".

-La RM N. 058-2011-PRODUCE, vigente desde el 26 de febrero del 2011, modifico el primer párrafo del Artículo $2^{\circ}$ de la $\mathrm{RM} \mathrm{N} \mathrm{N}^{\circ}$. 025-2011-PRODUCE, determinándose que el límite de captura establecido es de aplicación para las capturas provenientes de las embarcaciones pesqueras de mayor escala y será hasta la conclusión del régimen provisional de extracción del jurel y la caballa; se amplió el límite de captura a 45000 toneladas. Preciso que del límite de captura determinado en el presente régimen provisional, aplicable a las embarcaciones de mayor escala, queda por extraer $25236 \mathrm{t}$ de jurel.

-La RM Nº 099-2011-PRODUCE, vigente desde el 20 de marzo del 2011, suspendió las actividades de extracción del recurso jurel, a partir de las 00:00 horas del día siguiente de su publicación, en el marco del régimen provisional de extracción dispuesto por la RM Nº. 058-2011-PRODUCE.

-La RM No. 107-2011-PRODUCE, vigente desde el 25 de marzo del 2011, prorrogo la vigencia del régimen provisional de pesca del jurel y la caballa, establecido por la RM $N^{\circ}$. 025-2011-PRODUCE, hasta el 30 de abril de 2011, en todo el litoral peruano, de acuerdo con las razones expuestas en la parte considerativa de la resolución. Amplía el límite de captura aplicable a las capturas de las embarcaciones de mayor escala, en aplicación de la primera disposición final, complementaria y transitoria del Reglamento de Ordenamiento Pesquero (DS N. 011-2007-PRODUCE) hasta $55000 \mathrm{t}$ de jurel y caballa, y mantiene la vigencia de las demás disposiciones contenidas en la RM N $\mathrm{N}^{\circ}$. 025-2011, modificada por la RM Nº 058-2011-PRODUCE.
-La RM No. 124-2011-PRODUCE, vigente del 10 de abril del 2011, suspendió las actividades de extracción del jurel a partir de las 00:00 horas del día siguiente de su publicación, en el marco del régimen provisional de extracción dispuesto por la RM No. 025-2011-PRODUCE, modificado por la RM N ${ }^{\circ}$. 058-2011-PRODUCE y prorrogado por la RM Nº. 107-2011-PRODUCE.

-La RM No. 130-2011-PRODUCE, vigente desde 13 de abril del 2011, amplio el límite de las capturas provenientes de las embarcaciones pesqueras de mayor escala, hasta $62000 \mathrm{t}$ para el jurel, en el marco de lo dispuesto en el régimen provisional establecido por la RM N. ${ }^{\circ}$ 025-2011-PRODUCE.

-La RM N. 146-2011-PRODUCE, vigente desde el 28 de abril del 2011, prorrogo la vigencia del régimen provisional de extracción del jurel y la caballa, hasta el 30 de junio de 2011 en todo el litoral peruano, establecido por la RM N . 025-2011-PRODUCE.

-La RM N. ${ }^{\circ}$ 198-2011-PRODUCE, vigente del 29 de junio del 2011, estableció un límite de captura de 45000 t de jurel y caballa, a partir de las 00:00 horas del 1 de julio hasta las 24:00 horas del 30 de setiembre de 2011, en todo el litoral peruano.

-La RM N. 277-2011-PRODUCE, vigente desde el 01 de octubre del 2011, amplio el límite de captura del jurel y la caballa, establecido mediante la RM N. ${ }^{\circ}$ 198-2011-PRODUCE, a 75000 t, desde el día siguiente de su publicación hasta las 24:00 horas del 31 de diciembre de 2011, en todo el litoral peruano.

-La RM N. 034-2012-PRODUCE, vigente desde el 24 de enero del 2012, estableció el límite de captura del jurel y la caballa en 40000 y 20000 t respectivamente, correspondiente al primer trimestre del ańo 2012. Una vez cumplido el límite de captura para cada recurso, el Ministerio de la Producción suspendió las actividades extractivas por los días que restaron del periodo autorizado.

-La RM Nº 055-2012-PRODUCE, vigente desde el 03 de febrero del 2012, suspendió las actividades extractivas del jurel en todo el litoral peruano a partir de las 00:00 horas del día siguiente de su publicación. Los armadores que se encontraban pescando en el litoral peruano tuvieron un plazo de dos días para el desembarque. La RM N ${ }^{\circ}$. 133-2012-PRODUCE la dejo sin efecto.

-La RM N. 132-2012-PRODUCE, vigente del 22 de marzo del 2012, preciso que los alcances de la RM N ${ }^{\circ}$. 034-2012-PRODUCE en el límite de captura del jurel y caballa correspondiente al primer trimestre del año 2012, es para las actividades extractivas realizadas por la flota industrial pesquera. Lo cual indicaba que la suspensión de las actividades extractivas del recurso jurel, regulada en la RM Nº ${ }^{\circ}$ 055-2012-PRODUCE, no era de aplicación a la flota artesanal.

-La RM No. 133-2012-PRODUCE, vigente desde el 23 de marzo del 2012, estableció el límite de captura del jurel y caballa en 30 mil y 20 mil t respectivamente para la flota industrial, lo que correspondió al segundo trimestre del año 2012, (1 de abril al 30 de junio de 2012). Una vez cumplido el límite de captura para cada recurso, el Ministerio de la 
Producción suspendió las actividades extractivas por los días que restaban al periodo autorizado. Dejo sin efecto las disposiciones contenidas en la RM N. 055-2012-PRODUCE.

-La RM N. 142-2012-PRODUCE, vigente desde el 24 de marzo del 2012, modifico el Artículo $1^{\circ}$ de la RM N ${ }^{\circ}$. 133-2012-PRODUCE 23/03/2012, que estableció el límite de captura de jurel y caballa.

-La RM N. 173-2012-PRODUCE, vigente desde 15 de abril del 2012, estableció la cuota de captura total permisible para el jurel en $120000 \mathrm{t}$ correspondiente al año 2012. La cuota establecida comprendía las capturas efectuadas al amparo de la RM Nº. 034-2012-PRODUCE, precisada por la RM N ${ }^{\circ}$. 132-2012-PRODUCE y la RM $\mathrm{N}^{\circ}$. 133-2012-PRODUCE, modificada por la RM N . 142-2012_PRODUCE. En caso de cumplirse la cuota de captura establecida, el Ministerio de la Producción suspendería las actividades extractivas.

-La RM N. 191-2012-PRODUCE, del 19 de abril del 2012, suspendió las actividades extractivas del jurel a partir de las 00:00 horas del día 21 de abril de 2012, en todo el litoral peruano. En consecuencia, se prohibió transportar y comercializar jurel a partir del $4^{\circ}$ día desde la entrada en vigencia de la suspensión de sus actividades extractivas. Los armadores de embarcaciones pesqueras industriales que se encontraban capturando jurel en el litoral peruano tuvieron un plazo de dos días para efectuar el desembarque del volumen capturado.

\section{Medidas de seguimiento, control y vigilancia}

Un marco jurídico e institucional eficaz de ordenación pesquera exige que se apliquen medidas de seguimiento, control y vigilancia que garanticen el cumplimiento de las normas, principalmente en lo que se refiere a las actividades de pesca por parte de los administrados, incluyendo cuando procedan, programas de observadores, mecanismos de inspección y sistemas de vigilancia de buques (FAO 1995) que cumplan con evitar la pesca ilegal y la depredación de los recursos y, en general, que se asegure obtener los objetivos del ordenamiento pesquero establecidos.

En el caso de Perú, institucionalmente el Ministerio de Producción, a través del Viceministro de Pesquería, tiene la función de diseñar, establecer, ejecutar y supervisar, la política nacional y sectorial aplicable al sector de pesquería, la de dictar normas y lineamientos técnicos para la adecuada gestión de los recursos, así como para el otorgamiento, reconocimiento de derechos, la sanción, fiscalización y ejecución coactiva. Estas funciones se traducen a la labor del Estado en cuanto a regular el manejo integral y la explotación racional de los recursos hidrobiológicos; asimismo entre sus labores están el ser responsable del seguimiento y controlar las actividades pesqueras. Además, estas funciones incluyen el control de las embarcaciones de pesca mediante los sistemas de vigilancia satelital. Dichas acciones perfeccionan los tradicionales sistemas de control de las capturas que existen en los puertos donde los desembarcos permiten conocer y fiscalizar el cumplimiento de las cuotas de captura.

Si como efecto de la fiscalización de las actividades pesqueras se constata faltas a la normatividad vigente y se realiza un previo informe técnico sustentatorio, se apertura el procedi- miento administrativo sancionador a cargo de la Dirección General de Sanciones del Ministerio de la Producción. Este organismo deberá determinar si corresponde o no aplicar la sanción administrativa correspondiente, decisión que de ser impugnada por el armador y/o titular pesquero es sometida a revisión por parte del Consejo de Apelación de Sanciones, quien en su calidad de superior jerárquico hará una revisión de la legalidad del procedimiento seguido por la Dirección General de Sanciones.

Concluido el procedimiento sancionador en vía administrativa, el infractor deberá cumplir con las sanciones impuestas por el órgano sancionador, caso contrario se procederá a la ejecución coactiva de las mismas. Cabe señalar que, agotada la vía administrativa, el armador tiene la posibilidad de ejercer su derecho de defensa en la vía judicial, la cual hará una revisión del procedimiento administrativo a fin de que se hayan respetado las garantías del debido procedimiento.

Complementariamente a las responsabilidades del Ministerio de la Producción para formular, aprobar y supervisar las políticas de alcance nacional aplicables a las actividades extractivas y productivas del sector pesquería, y dictar normas de alcance nacional y supervisar su cumplimiento, el Ministerio de Defensa a través de la Dirección General de Capitanías y Guardacostas de la Marina de Guerra del Perú ejerce la autoridad marítima, fluvial y lacustre. Es decir, esta dirección es responsable de normar y velar por la seguridad de la vida humana, la protección del medio ambiente y sus recursos naturales, así como reprimir todo acto ilícito, ejerciendo el control y vigilancia de todas las actividades que se realizan en el medio acuático, de esta manera contribuye al desarrollo nacional.

\section{Hacia la aplicación del enfoque ecosistémico}

También se acepta que el concepto tradicional del ordenamiento pesquero basado en un enfoque dirigido a una sola especie ha de cambiar hacia un concepto ecosistémico, reconociendo que, en general, la actividad extractiva no sólo afecta a la población objetivo, sino a todos los elementos del ecosistema.

Por lo tanto, se requiere un enfoque diferente, cuyo objetivo fundamental sea el aprovechamiento sostenible del ecosistema. Es conveniente que este planteamiento que dio origen a la aplicación del enfoque ecosistémico a la pesca en general, sea incorporado al ordenamiento del jurel y caballa. Sobre la base del enfoque ecosistémico a la pesca se podrá comprender la planificación, desarrollo y ordenación de la pesca de estas especies, de un modo que la actividad satisfaga las múltiples necesidades de nuestra sociedad actual, sin poner en riesgo la posibilidad de que las generaciones futuras se beneficien también de los bienes y servicios que pueden obtenerse de la integridad del ecosistema marino.

En este enfoque, la estrategia para incluir la pesquería del jurel y la caballa demanda que el sistema de ordenamiento actual incorpore algunas necesidades o requerimientos, como obtener mejor información para formular las políticas y los planes de ordenamiento. Estos planes deben considerarse como la base técnica de los reglamentos de ordenamiento pesquero en la perspectiva jurídica, y los planes de ordenación en la descripción de la biología de las especies que son objeto de nuestra atención en el presente caso, y en su interrelación con el ecosistema. Es imperativo el establecimiento de objetivos e indicadores ope- 
racionales para la pesquería de jurel y caballa, en tanto estas especies continúen tratándose como integrantes de una actividad.

Una vez establecidos los objetivos operacionales, será necesario determinar las medidas de ordenamiento, las cuales podrán darse sobre la base de las medidas vigentes, pero orientadas a mantener o restablecer la estructura y funcionamiento del ecosistema.

Finalmente, ratificamos que una buena base técnica para avanzar hacia un ordenamiento pesquero basado en el enfoque ecosistémico a la pesca lo viene proporcionando el sistema de observación del ambiente, los recursos vivos y sus pesquerías, que el IMARPE desarrolla desde hace casi 50 años y se evidencia en la presente publicación, que comprende muchos de los aspectos biológicos y pesqueros del jurel, así como del ambiente en el cual se desarrolla.

\section{Conclusiones}

La actividad pesquera es una fuente importante de suministros para la seguridad alimentaria de la población peruana y proporciona beneficios económicos y sociales para la nación. Uno de los principales recursos hidrobiológicos de la pesca marina es el jurel que ha representado en los últimos ańos cerca del $30 \%$ de la captura total de especies marinas con destino al consumo humano directo. Estas consideraciones obligan a que la gestión pesquera del recurso sea efectiva y exitosa. Se entiende como gestión exitosa el mantener la sostenibilidad de la pesquería, la conservación del recurso y el mantenimiento de su aporte a la producción de consumo humano directo. Correspondiendo a este interés nacional, se ha establecido un sistema de ordenamiento y regulación de la pesca de jurel que se fundamenta en los principios de las leyes de Aprovechamiento Sostenible de los Recursos Naturales y General de Pesca, y se hace efectivo con el Reglamento de Ordenamiento Pesquero de los recursos jurel y caballa vigente.

En el presente caso sobre el ordenamiento pesquero del jurel, se demuestra que la legislación nacional es adecuada. Para ello se tiene en consideración que abarca un conjunto de principios normativos y reglamentos nacionales. Estos principios normativos y reglamentos definen con claridad los objetivos del ordenamiento y las medidas de gestión de la pesca del jurel peruano T. murphyi, como también la consiguiente protección del ambiente asociado a los recursos en explotación. El reglamento respectivo incluye medidas como la fijación de cuotas de capturas permisibles con un único destino para el consumo humano, las temporadas de pesca, el esfuerzo pesquero, los métodos de pesca, tamaño mínimo de malla de las redes, el tamaño mínimo de captura de los ejemplares de jurel. Asimismo, entre las medidas incluidas está el establecimiento de otras normas que son en el presente necesarias para la conservación y explotación racional de los recursos en general y de esta especie en particular. Además, hace posible la definición de más medidas, de acuerdo a la condición o estado del recurso hidrobiológico. Muchas de las normas que se aplican actualmente en la pesquería de la especie han sido descritas en el presente trabajo, cuya finalidad ha sido mostrar que el esquema de gestión del jurel cumple con el propósito de mantener al recurso con la capacidad de proporcionar capturas sostenidas destinadas al consumo humano, así como la viabilidad económica y social de su desarrollo.
Más aún, se puede afirmar que mediante el establecimiento y aplicación de este sistema de ordenamiento se cumple con el derecho internacional, el cual está reflejado en las disposiciones pertinentes de la Convención de las Naciones Unidas sobre el Derecho del Mar de 1982. Para los estados ribereños que tienen aguas bajo jurisdicción nacional, en ese documento se establece el privilegio de ejercer sus derechos de soberanía para los fines de exploración, explotación, conservación y gestión de la pesca de los recursos y la conservación de los recursos marinos vivos sobre los que la pesca tiene un impacto.

Si bien con este sistema de gestión de la pesca se demuestra el éxito de su implementación a nivel nacional, ha existido y existe permanentemente el riesgo potencial del desarrollo indiscriminado de la pesca sobre esta unidad de stock de jurel en alta mar, lo cual puede desestabilizar su equilibrio poblacional y, por lo tanto, afectar la pesquería peruana. Por esta razón, el Perú ha participado activamente en la conformación de una entidad de ordenamiento pesquero para el alta mar, la OROP del Pacífico Sur (Organización Regional de Ordenación Pesquera del Pacífico Sur), la cual es una organización intergubernamental que debe estar permanentemente comprometida con la conservación a largo plazo, el uso sostenible de los recursos pesqueros en el océano Pacífico Sur y la protección de los ecosistemas marinos en los que dichos recursos se producen. Estos principios son reconocidos por la convención al igual que la existencia de consideraciones económicas y geográficas, además de las necesidades especiales de los estados y sus comunidades costeras, en relación con el desarrollo de la conservación y la gestión sostenible de los recursos pesqueros, así como es reconocida la explotación equitativa de esos recursos, por la cual se acordó establecer dicha organización.

\section{Literatura citada}

Constitución Política del Perú. 1993. Congreso de la República del Perú. 1993. <http://www.tc.gob.pe/legconperu/constitucion.html>. Acceso 20 de junio del 2013

DL Nº 25977. 1992. Ley General de Pesca. Congreso de la República del Perú. 21 de de diciembre de 1992. <http://www2.produce.gob. pe/dispositivos/publicaciones/2013/rm077-2013-produce.pdf $>$. Acceso 16 de junio del 2013

DS Nº. 012-84-PE. 1984. Reglamento para la Operación de Buques Pesqueros de Arrastre. LEX-FAOC018459. 19/09/1984. Acceso 24 de agosto 2013.

DS Nº. 017-92-PE. 1992. Ministerio de Pesquería. Prohíben la pesca con el uso de métodos que modifiquen las condiciones bioecológicas del medio marino en la zona adyacente a la costa, comprendida entre las cero y cinco millas marinas. Revista de Legislación y Jurisprudencia. Normas Legales, Tomo 196, setiembre 1992. pagina 212.

DS Nº ${ }^{\circ}$ 012-2001-PE. 2001. Aprueban el Reglamento de la Ley General de Pesca, Ministerio de la Producción. 13 de marzo de 2001. El Peruano Normal Legales: 199905-199921

DS $N^{\circ}$. 001-2002-PRODUCE. 2002. Establecen que los recursos sardina, jurel y caballa serán destinados al consumo humano directo. 06 de setiembre 2002. El Peruano. Normas Legales: 229522-229523.

DS N. 031-2003-PRODUCE. 2003. Establecen Marco Provisional para la Implementación del Sistema de Seguimiento Satelital. 04 de noviembre 2003. El Peruano Normas Legales: 254393-254394.

DS Nº 011-2007-PRODUCE. 2007. Aprueban Reglamento de Ordenamiento Pesquero de Jurel y Caballa. 13de abril de 2007. El Peruano Normal Legales: 343399-343402

DS N ${ }^{\circ}$. 022-2009-PRODUCE. 2009. Decreto Supremo que establece medidas de ordenamiento para la extracción comercial a mayor escala de recursos hidrobiológicos tranzonales (jurel y caballa) en la alta mar. 05 de julio 2009. El Peruano Normas Legales: 398635-398638.

FAO. 1995. Código de Conducta para la Pesca Responsable. 1995. Roma. FAO. 46 p.

FAO. 1999. La ordenación pesquera. Orientaciones Técnicas para la Pesca Responsable N. ${ }^{\circ}$ 4. Roma. FAO. 81 p. 
Ley Nº. 26821. 1997. Aprueba Ley Orgánica para el aprovechamiento. Sostenible de los recursos naturales. Congreso de la República del Perú. 26 de junio de 1997. <http://www2.produce.gob.pe/RepositorioAPS/1/jer/PROPESCA_OTRO/marco-legal/1.3.\%20Ley26821. pdf>. Acceso 15 de junio del 2013.

RM Nº. 0171-74-PE. 1974. Ministerio de Pesquería. 1974. Prohíben extracción de especies hidrobiológicas cuya longitud sea inferior a lo autorizado. Revista de Legislación y Jurisprudencia Normas Legales, Tomo 70, enero- marzo 1974: pagina 145.

RM N ${ }^{\circ}$. 026-2010-PRODUCE. 2010. Establecen régimen provisional para la extracción de los recursos jurel y caballa en todo el litoral peruano. 03 de febrero 010. El Peruano Normas Legales: 412689-412690.

RM N. 027-2010-PRODUCE. 2010. Establecen régimen de pesca exploratoria de recursos pelágicos de oportunidad asociados al evento El Niño en todo el litoral. 03 de febrero 010. El Peruano Normas Legales: 412690-412692.

RM N. 096-2010-PRODUCE. 2010. Establecen Régimen Provisional para la extracción de los recursos jurel y caballa en todo el litoral peruano. 18 de abril 2010. El Peruano Normas Legales: 417356-417357.

RM Nº. 097-2010-PRODUCE. 2010. Establecen Régimen de Pesca Exploratoria de recursos pelágicos de oportunidad asociados al evento El Niño en todo el litoral peruano. 18 de abril 2010. El Peruano Normas Legales: 417357-417359.

RM Nº. 202-2010-PRODUCE. 2010. Establecen Régimen Provisional para la extracción de los recursos jurel y caballa en todo el litoral peruano. 18 de agosto de 2010. El Peruano Normas Legales: 423977-423978

RM N. 025-2011-Produce. 2011. Establecen Régimen Provisional para la extracción de los recursos jurel y caballa en todo el litoral peruano. 26 de enero 2011. El Peruano Normas Legales: 434925-434927.

RM N$^{\circ}$. 035-2011-PRODUCE. 2011. Suspenden actividades de extracción y recepción del recurso jurel en el marco establecido mediante R.M. N. ${ }^{\circ} 025-2011-P R O D U C E .06$ de febrero 2011. El Peruano Normas Legales: 435774-435775.

RM No. 039-2011-PRODUCE. 2011. Modifican la R.M. No 035-2011-PRODUCE mediante la cual se dispuso suspender las actividades de extracción y recepción del recurso jurel. 09 de febrero de 2011. El Peruano Normas Legales: 435847-435848.

RM N$^{\circ}$. 058-2011-PRODUCE. 2011. Modifican R.M. N. ${ }^{\circ} 025-2011$-PRODUCE en lo referente al límite de captura establecido en el Régimen Provisional para la extracción de los recursos jurel y caballa. 26 de febrero 2011. El Peruano Normas Legales: 437661-437662.

RM Nº. 099-2011-PRODUCE. 2011. Suspenden actividades de extracción de los recursos jurel, anchoveta y anchoveta blanca. 20 de marzo 2011. El Peruano Normas Legales: 439295-439296.

RM Nº. 107-2011-PRODUCE. 2011. Prorrogan vigencia del Régimen Provisional de pesca de los recursos jurel y caballa establecida mediante RM No. 025-2011-PRODUCE. 25 de marzo 2011. El Peruano Normas Legales: 439597-439598.
RM N. 124-2011-PRODUCE. 2011. Suspenden actividades de extracción del jurel. 10 de abril de 2011. El Peruano Normas Legales: 440684440686

RM Nº. 130-2011-PRODUCE. 2011. Amplían límite de capturas provenientes de las embarcaciones pesqueras de mayor escala, en el marco establecido mediante R.M. N. ${ }^{\circ}$ 025-2011-PRODUCE. 13 de abril 2011. El Peruano Normas Legales: 440876-440877.

RM N. 146-2011-PRODUCE. 2011. Prorrogan vigencia del Régimen Provisional para la extracción de los recursos jurel y caballa establecido por R.M. N. ${ }^{\circ}$ 025-2011-PRODUCE. 28 de abril 2011. El Peruano Normas Legales: 441665-441666.

RM N ${ }^{\circ}$. 198-2011-PRODUCE. 2011. Establecen límite de captura de recursos jurel y caballa. 29 de junio 2011. El Peruano Normas Legales: 445535-445536.

$\mathrm{RM} \mathrm{N}^{\circ}$. 034-2012-PRODUCE. 2012. Establecen límite de captura de recursos jurel y caballa, correspondiente al primer trimestre del ańo 2012 . 24 de enero 2012. El Peruano Normas Legales: 459721-459722.

RM N ${ }^{\circ}$. 055-2012-PRODUCE. 2012. Suspenden actividades extractivas del recurso jurel en todo el litoral. 03 de febrero 2012. El Peruano Normas Legales: 460291.

RM Nº. 132-2012-PRODUCE. 2012. Precisan alcances de la R.M. N. ${ }^{\circ}$ 034-2012-PRODUCE en el extremo referente al límite de captura de los recursos jurel y caballa correspondientes al primer trimestre del año 2012. 22 de marzo 2012. El Peruano Normas Legales: 462911.

RM Nº. 133-2012-PRODUCE. 2012. Establecen límite de captura de los recursos jurel y caballa para la flota industrial pesquera, correspondiente al segundo trimestre del ańo 2012. 23 de marzo 2012. El Peruano Normas Legales: 462958.

RM N$^{\circ}$. 142-2012-PRODUCE. 2012. Modifican la R.M. N. ${ }^{\circ}$ 133-2012-PRODUCE, que estableció el límite de captura de los recursos jurel y caballa para el segundo trimestre de 2012. 24 de marzo 2012. El Peruano Normas Legales: 463015-463016.

RM N. 173-2012-PRODUCE. 2012. Establecen cuota de captura total permisible para el recurso jurel correspondiente al ańo 2012. 15 de abril 2012. El Peruano Normas Legales: 464166-464167.

RM N ${ }^{\circ}$. 191-2012-PRODUCE. 2012. Suspenden actividades extractivas del recurso jurel efectuadas por la flota industrial, en todo el litoral peruano, a partir del 21 de abril de 2012. 20 de abril 2012. El Peruano Normas Legales: 464560-464561.

RM No. 277-2011-PRODUCE. 2011. Amplían límite de captura de los recursos jurel y caballa establecido mediante RM No. 198-2011-PRODUCE. 01 de octubre 2011. El Peruano Normas Legales: 450954-450955. 
